\title{
Outcome of surgical resection for hilar cholangiocarcinoma
}

\author{
Magdy A Sorour, MD; Mohamed T El-Riwini, MD
}

GIT Surgery Unit, General Surgery Department, University of Alexandria, Egypt.

Background: Hilar cholangiocarcinoma (HC) is an adenocarcinoma of the extrahepatic biliary tree arising from the main left or right hepatic ducts or their confluence. HC is still considered to be a disease which is difficult to treat or to cure. Its prognosis is very poor and the only curative treatment is complete resection with a negative surgical margin. Preoperative evaluation of the tumor is important in order to evaluate resectability and the extent of surgery. Surgical resection involves either local resection, local excision with caudate lobectomy or major hepatic resection with caudate lobectomy. The aim of this work was to study the feasibility and outcome of surgical resection for hilar cholangiocarcinoma.

Methods: This study was carried out on 72 patients with radiological evidence suggesting resectable hilar cholangiocarcinoma presented to the Gastrointestinal Surgery Unit, Main Alexandria University Hospital during the period from March 2006 till January 2013. All patients' presentations, laboratory and radiological data, surgical procedures, complications, follow-up and survival data were collected.

Results: Between March 2006 and January 2013; 72 patients (56 males and 16 females) were admitted for resection of a radiologically suspected resectable hilar cholangiocarcinoma. All patients presented with obstructive jaundice of varying degrees. The tumors were BismuthCorlette type I in 36 patients, type II in 24 patients, type IIIa in 5 patients and type IIIb in 7 patients. All patients underwent surgical resection. Forty-six patients underwent bile duct resection with hepaticojejunostomy and regional lymph node dissection. Fourteen patients underwent bile duct resection and lymph node dissection with caudate lobectomy. Three patients underwent right hepatectomy, two patients underwent extended right hepatectomy, four patients underwent left hepatectomy and three patients underwent extended left hepatectomy; all with hilar bile duct resection, caudate lobectomy and regional lymph node dissection. Complete resection (R0) was achieved in 65 patients (90.3\%), while 7 patients (9.7\%) had incomplete resection. All over 9 patients (12.5) developed recurrence. The 3 and 5-years overall survival rate for all patients, using the Kaplan-Meier actuarial curve, were $51.4 \%$ and $34.7 \%$ respectively.

Conclusion: Cholangiocarcinoma remains a devastating disease. Most patients have unresectable tumors at the time of diagnosis and have a dismal prognosis. Complete resection is the only treatment that offers any hope of long-term survival but is possible in few patients. Furthermore, even after resection, disease recurrence is common. Adjuvant therapy has not been shown to have a role in this disease.

Key words: Bile duct cancer, hilar cholangiocarcinoma, Klatskin tumor, staging, surgical resection.

\section{Introduction:}

Hilar cholangiocarcinoma (HC) is an adenocarcinoma of the extrahepatic biliary tree arising from the main left or right hepatic ducts or their confluence. This tumor has been referred to as Klatskin tumor after Dr. Gerald
Klatskin published his paper in $1965 .{ }^{1}$ About two-thirds of extrahepatic bile duct (EBD) cancers arise at the hepatic hilum (Klatskin tumor) with one third arising from the distal common bile duct.2,3 The diagnosis of $\mathrm{HC}$ should be suspected in patients with painless 
jaundice whose CT scan demonstrates dilated intrahepatic bile ducts with a normal gallbladder and extrahepatic biliary tree. ${ }^{4}$

$\mathrm{HC}$ is still considered to be a disease which is difficult to treat or to cure 5 . Its prognosis is very poor and the only curative treatment is complete resection with a negative surgical margin.2,3,6,7 Most patients with cholangiocarcinoma present with advanced disease that is not amenable to surgical treatment. ${ }^{8}$ Palliative resection, surgical bypass procedures, and various types of intubation and drainage procedures are associated with 3-year survival rates from $0 \%$ to $4 \% .{ }^{4}$ Adjuvant therapy (chemotherapy and radiation therapy) has not been shown clearly to reduce recurrence risk ${ }^{8}$.

In the extrahepatic ducts, three distinct macroscopic subtypes of cholangiocarcinoma are well described: sclerosing, nodular, and papillary. ${ }^{9}$ Sclerosing tumors are the most common subtype and are more common at the hilus than in the distal bile duct. Sclerosing tumors are very firm and cause an annular thickening of the bile duct, often with diffuse infiltration and fibrosis of the periductal tissues and can form an associated mass. Nodular tumors are characterized by a firm, irregular nodule of tumor that projects into the lumen of the duct. Features of both types are often seen, hence the frequently used description nodular sclerosing. The papillary variant accounts for approximately $10 \%$ of all cholangiocarcinomas and, although occasionally seen at the hilus, is more common in the distal bile duct. ${ }^{9}$ These tumors are soft and friable and may be associated with little transmural invasion. Tumor fragments may be present in the distal bile duct, resulting in intermittent jaundice. Recognition of this variant is important because it is more often resectable and may have a more favorable prognosis than the other types, although this has not been proved definitively. ${ }^{9}$ It can be divided further into papilloma type, intraductal growing type, mucin-producing type, and cystic type. ${ }^{10,11}$

Ninety percent of patients with HC have painless jaundice, $10 \%$ have cholangitis, and 56\% have systemic symptoms such as malaise, abdominal discomfort, nausea, anorexia, and weight loss. ${ }^{12,13}$ Preoperative evaluation of the tumor is important in order to evaluate resectability and the extent of surgery.2,3,6 In patients with hilar cholangiocarcinoma, evaluation must address four critical components of resectability: level and extent of tumor within the biliary tree, vascular invasion, hepatic lobar atrophy, and distant metastatic disease. Several methods are proposed to evaluate tumor extension. Bismuth-Corlette classification has been used to define the longitudinal tumor extension. ${ }^{14}$ Resectability of the tumor can be evaluated by the Blumgart T-staging system combined with the AJCC cancer staging system (American Joint Committee on Cancer). ${ }^{15}$ Assessment of the lateral spread and soft tissue extension can be evaluated based on the TNM staging system. 16,17 Lymph node metastasis is a significant factor helping to determine patient outcome after surgery for HC. ${ }^{18,19}$

MRI, CT, endoscopic retrograde cholangiography (ERC), and, perhaps, endoscopic ultrasound (EUS) are used most frequently to diagnose and stage $\mathrm{HC}$. The Bismuth-Corlette type I tumor is defined by the presence of a lesion confined below the confluence of the right and left hepatic ducts. This type of tumor can be treated with segmental resection of the EBD and regional lymph node dissection. Hepatectomy is unnecessary if the resection margin is microscopically confirmed to be negative by frozen pathology during surgery. ${ }^{20}$ Bismuth type II tumors extend to the confluence of the right and left hepatic ducts. Bismuth type II tumor can be treated by bile duct resection with hepaticojejunostomy and regional lymph node dissection. Caudate lobectomy is mandatory when the tumor infiltrates caudate bile duct branches. ${ }^{20,21}$ A type IIIa tumor extends to the bifurcation of the right hepatic duct, and a type IIIb tumor extends to the bifurcation of the left hepatic duct. Hilar bile duct resection with hemihepatectomy including the caudate lobectomy and regional lymph node dissection is the standard surgical method for type III tumor. ${ }^{20-22}$ Type IV tumors extend to the bifurcation of both 
the right and left hepatic ducts and have been generally regarded as inoperable except for liver transplantation; multicentric tumors are also included in this category. ${ }^{17}$

A preoperative $\mathrm{T}$ staging system, defined by biliary tumor extent, the presence or absence of portal vein involvement, and the presence or absence of hepatic lobar atrophy, may be useful for predicting resectability and the likelihood of finding metastatic disease. 23,24

The aim of this work was to study the feasibility and outcome of surgical resection for hilar cholangiocarcinoma.

\section{Methods:}

Between March 2006 and January 2013; 72 patients (56 males and 16 females) with radiological evidence suggesting resectable hilar cholangiocarcinoma were admitted in the Gastrointestinal Surgery Unit, Main Alexandria University Hospital for surgical resection. If unresectability was identified at laparotomy, palliative options were performed and these patients were excluded from the study.

After approval of local ethics committees of both the General Surgery Department and the Alexandria Faculty of Medicine, all patients included in the study were informed well about the operative procedure and the possible complications and an informed written consent was obtained from every patient before carrying the procedure.

All patients were subjected to the following Preoperative assessment:

Clinical 1) Complete history taking. 2) Thorough clinical examination

Laboratory work-up. 1) Routine laboratory studies 2) Bilirubin, alkaline phosphatase, CA19.9, CEA.

Imaging. 1) Abdominal duplex ultrsonography 2) Multi-slice CT (MSCT) abdomen. CT angiography whenever indicated. 3) MRI with magnetic resonance cholangiopancreatography (MRCP). 4) Endoscopic ultrasound (EUS): whenever indicated. 5. Endoscopic retrograde cholangiography (ERC): whenever indicted. 6) Percutaneous transhepatic cholangiography (PTC): as an alternative to ERC when endoscopy is unsuccessful or technically unfeasible. 7) Laparoscopy.

Cytology. 1) Bile duct brushing cytology at the time of ERC and PTC. 2) Peritoneal washing at the time of laparoscopy. 3) Fine needle aspiration cytology at the time of endoscopic ultrasound

Criteria of unresectability23,25

Patient factors

- Medical unfitness

- Advanced hepatic cirrhosis

Local tumor-related factors

- Invasion of the secondary biliary radicles bilaterally

- Encasement or occlusion of the main portal vein proximal to its bifurcation

- Any two combinations (one unilateral and one contralateral) of hepatic lobe atrophy, portal vein branch encasement or occlusion and secondary biliary radicles involvement.

\section{Metastatic Disease}

- Histologically proven metastases to N2 lymph nodes (peripancreatic, periduodenal, celiac, superior mesenteric, or posterior pancreaticoduodenal lymph nodes)

- Lung, liver, or peritoneal metastases

Metastatic disease to cystic duct, pericholedochal, hilar or portal lymph nodes (i.e., within the hepatoduodenal ligament) did not necessarily constitute unresectability.

Complications related to biliary tract obstruction or previous biliary intervention (ie, cholangitis, pancreatitis), if present, were treated before surgery. Routine biliary drainage of jaundiced patients, not previously stented and without cholangitis, was not performed if an operation could be performed in a timely fashion (within one week). Preoperative portal vein embolization was not used as there was no lobar atrophy in our series.

Biopsy material from the referring center was re-examined. In patients unfit for surgery and those with advanced disease, biopsy confirmation was performed, if not done previously. However, when the imaging studies suggested a potentially resectable $\mathrm{HC}$, histologic confirmation of malignancy was not performed. 
Surgical resection: its extent was determined by preoperative and intraoperative data. Because cholangiocarcinoma is known to spread along the wall of the bile ducts and because the caudate lobe is a frequent site of tumor recurrence following extrahepatic duct resection, a resection that includes the caudate lobe was performed. Tumor extension into only the right or the left lobe was resected by an en bloc extended left or right hepatectomy.

The resection was performed through a bilateral subcostal incision. A self-retaining retractor was used to provide adequate exposure. Following thorough exploration to confirm the absence of peritoneal disease, a complete retroduodenal and retropancreatic lymph node dissection was performed by mobilizing the entire right colon, duodenum, and pancreatic head in a lateral to medial rotation. The node-bearing tissue was dissected by removing the anterior aspect of Gerota fascia of the right kidney, then completely clearing all tissue off the anterior aspects of the right renal vein, right renal artery, inferior vena cava, and aorto-caval groove.

After completion of the retroduodenal and retropancreatic dissection, the gastrohepatic and gastroduodenal ligaments were divided along the superior edge of the stomach and duodenum. The cystic duct and cystic artery were identified. The cystic artery was ligated and divided. The gallbladder was completely dissected free from the gallbladder bed but left attached to the bile duct by the cystic duct. The gastroduodenal artery, the proper hepatic artery, and the portal vein were dissected circumferentially from the gastrohepatic ligament and the bile duct. This dissection was carried from caudad to cephalad toward the confluence of the left and right portal veins and the liver plate. Portal vein involvement was considered present if the tumor contacted and either distorted or narrowed the vein, or if the vein was encased or occluded. After the bile duct tumor was dissected from the anterior surface of the portal vein, the distal common bile duct at the superior aspect of the duodenum was divided and suture-ligated. The common bile duct, gallbladder, and node-bearing porta hepatis tissue were then reflected in an anterior and cephalad direction to complete the posterior dissection along the right and left bile ducts. A circumferential dissection of the right and left bile ducts was performed if tumor was isolated to the confluence of the right and left bile duct (not extending up into either the right or left duct). The right and left bile ducts were then divided sharply at least $1 \mathrm{~cm}$ proximal to the tumor. The surgical specimen was removed and included the distal right and left bile ducts, the common hepatic duct, the common bile duct with the attached gallbladder, and the regional node-bearing tissue. The specimen was immediately evaluated using frozensection studies on the right and left bile ducts to confirm tumor-negative margins. At that point, the gastroduodenal artery, proper hepatic artery, left and right hepatic arteries, portal vein, and inferior vena cava had been "laid bare" Figure (1).

Theresection was completed by performing a caudate lobectomy. The capsule of the caudate lobe was scored with electrocautery and then the caudate lobe was dissected free from the vena cava using an ultrasonic dissecting instrument. Small vessels within the parenchyma of the caudate lobe were clipped and larger vessels were ligated. The removal of the caudate lobe was completed by dissecting the liver parenchyma free from the anterior aspect of the retrohepatic vena cava. The small venous branches draining directly from the caudate lobe into the vena cava were identified, suture-ligated, and divided until the caudate lobe had been removed.

Biliary-enteric drainage was reestablished using a Roux-en-Y jejunal loop. The right and left bile ducts were individually anastomosed end-to-side to the jejunal loop using fullthickness interrupted 4-0 absorbable vicyl sutures Figure (2). The operation was completed by placing two tube drains near the base of the liver. The abdominal wall was closed in layers.

Incases wherethehilarcholangiocarcinoma extended directly into the right or left bile duct, an extended right or left hepatectomy was performed en bloc with the extrahepatic bile 
duct, gallbladder, and regional node-bearing tissue Figures(3,4). Only a single Roux-en-Y hepaticojejunostomy was performed.

A nasogastric tube was placed during the operation. We generally remove the nasogastric tube on the 1st postoperative day. On postoperative day 5, we removed the tube drains. All patients received a perioperative dose of a third-generation cephalosporin antibiotic and then received scheduled intravenous dosages of this antibiotic during the following 5 days after surgery.

In patients who had a bile leak from a biliary-enteric anastomosis that was drained by the surgically placed drains, the drains were not removed on postoperative day 5 but were kept in place until the biliary fistula had closed. If a fluid collection was demonstrated, percutaneous drainage of the bile collection was performed.

Postoperative data: i) Histopathology of the resected tumor. ii) Hospital stay. iii)Early and late post-operative complications.

Patient follow-up: Patients were followed regularly in an outpatient setting every one to six months. The sites of disease recurrence (local and distant) were determined from imaging studies, including CT and MRI. Survival (overall and disease-free survival) were also estimated.

Data were presented with numbers, percentage, arithmetic mean (X) and standard deviation (SD) and were analyzed with SPSS (version 16) statistical software. Disease free survival curve and overall survival curve were estimated using the Kaplan-Meier method. $P$ values less than 0.05 were considered to be statistically significant. A multivariate Cox proportional hazards model was used to evaluate which factors demonstrated an independent effect on postoperative estimated survival.

\section{Results:}

Between March 2006 and January 2013; 72 patients were admitted for resection of a radiologically suspected resectable hilar cholangiocarcinoma. Their mean age at diagnosis was $57.76 \pm 10.23$ years (ranged from 41 to 69 years).
All patients presented with obstructive jaundice of varying degrees. Other presentations are shown in Table(1). Fiftyeight patients were Child grade $\mathrm{A}$ and 14 patients were Child grade B. Sixty-five patients had non-cirrhotic livers and 7 patients had cirrhotic liver.

Data regarding patients' age, sex, tumour extension, extent of resection, operative time, intra-operative bleeding (as recorded by the anaesthesiologist), histological grade, lymph node status, hospital stay (from the time of operation to discharge) and postoperative complications are shown in Tables $(2,3,4)$.

Tumors were Bismuth-Corlette type I in 36 patients, type II in 24 patients, type IIIa in 5 patients and type IIIb in 7 patients. All patients underwent surgical resection. Fortysix patients underwent bile duct resection with hepaticojejunostomy and regional lymph node dissection. Fourteen patients underwent bile duct resection and lymph node dissection with caudate lobectomy. Three patients underwent right hepatectomy, two patients underwent extended right hepatectomy, four patients underwent left hepatectomy and three patients underwent extended left hepatectomy; all with hilar bile duct resection, caudate lobectomy and regional lymph node dissection. In the 12 patients who underwent major hepatic resections, the residual liver volume was more than $30 \%$ in 10 patients and less than 30 in two patients.

Operative estimated blood loss was greater in patients who underwent hepatic resection (26 patients; caudate lobectomy in 14 and major hepatectomy in 12). In addition, all patients who underwent major hepatic resection required blood transfusion.

The number of lymph nodes removed was fewer than 12 in 17 patients and equal or more than 12 in 55 patients. The median number of lymph nodes removed was 17 (range 7-32). Lymphatic invasion was negative in 40 patients and positive in 32 patients.

The lymph node ratio (LNR), defined as the ratio of the number of lymph nodes harboring metastases to the total number of lymph nodes removed was less than 0.2 in 57 patients and equal or more than 0.2 in 15 
patients.

Complete resection (R0) was achieved in 65 patients, while 7 patients had incomplete resection. Two patients died of hepatic failure. Their ages were 59 and 66 years. Death occurred at 7 and 35 days following extended left hepatectomy and right hepatectomy.

All over 9 patients (12.5) developed recurrence. One patient underwent revisional hepaticojejunostomy following the development of a left hepatic duct stricture 1 year after extended right hepatectomy and bile duct excision. Subsequent histological examination showed this to be due to tumour recurrence, although the patient was alive and symptom free 15 months after this reintervention.

For all 72 patients, the overall estimated survival rate was $51.4 \%$ at three years and $34.7 \%$ at five years. Forty patients with no lymph node metastases had a five-year estimated survival of 55\% in comparison to a five-year survival of $9.4 \%$ for the 32 patients with lymph node metastases $(p=0.015)$. Fifty-seven patients with LNR less than 0.2 had a five-year estimated survival of $43.9 \%$ in comparison to a five-year survival of $0 \%$ for the 15 patients with LNR equal or more than $0.2(\mathrm{p}=0.005)$.

In the multivariate analysis, the following factors were independent prognostic survival factors (1) Child's grade, (2) residual disease, (3) lymph node metastasis and (4) lymph node ratio (LNR). Other factors, such as operative time, intra-operative bleeding, histological grade, and number of lymph nodes removed were not significant predictors of estimated survival.

\section{Discussion}

This study included 72 patients with a radiologically suspected resectable hilar cholangiocarcinoma. The first sign of hilar cholangiocarcinoma in the studied patients was jaundice. Many patients had one or more nonspecific complaints, such as abdominal pain, general malaise, anorexia, and weight loss. Although most patients eventually became jaundiced, those with ipsilateral or segmental involvement may have abnormal liver function tests and even pruritus without jaundice. ${ }^{26}$ Cholangitis is rarely a presenting feature in patients with cholangiocarcinoma in the absence of prior biliary intubation. Most patients with hilar strictures and jaundice have cholangiocarcinoma. However, alternative diagnoses can be expected in 10 to $15 \%$ of patients, the most common of which are gallbladder carcinoma, Mirizzi syndrome, and idiopathic benign focal stenosis (malignant masquerade). ${ }^{26}$

Benign strictures of the proximal biliary tree are uncommon, and hilar cholangiocarcinoma must remain the leading diagnosis until definitively disproved. In most cases, this cannot be done without exploration. Relying on the results of percutaneous needle biopsy or biliary brush cytology is dangerous, because the results are often misleading, and one may miss the opportunity to resect an early cancer.

In the present study, tumors were BismuthCorlette type I in 36 patients, type II in 24 patients, type IIIa in 5 patients and type IIIb in 7 patients. Bismuth-Corlette classification has been used to define the longitudinal tumor spread in one dimension along the bile duct and it does not incorporate radial tumor growth. ${ }^{27}$ Surgical candidates cannot be determined solely by this classification, and it is not indicative of survival. ${ }^{28}$ Another aspect to consider is that longitudinal spread pattern of a tumor can be related to gross morphology. ${ }^{29}$ Papillary tumors frequently present with long-range mucosal spread, while infiltrating tumors tend to show subepithelial extension. The subepithelial infiltration may readily be depicted on $\mathrm{CT}$ or MRI by showing thickening or increased enhancement of the ductal wall, but the mucosal spread may hardly be visible on CT or MRI. Therefore, determination of longitudinal spread must be made more cautiously when a papillary or polypoid tumor is seen on imaging. Abe $M$ et $\mathrm{al}^{30}$ presented an illustrative case in which the utility of choledocoscopy is demonstrated. In that case, cholangiography showed a polypoid tumor in the middle $\mathrm{CBD}$, but choledochoscopy demonstrated multifocal superficial spreading tumors along the entire 


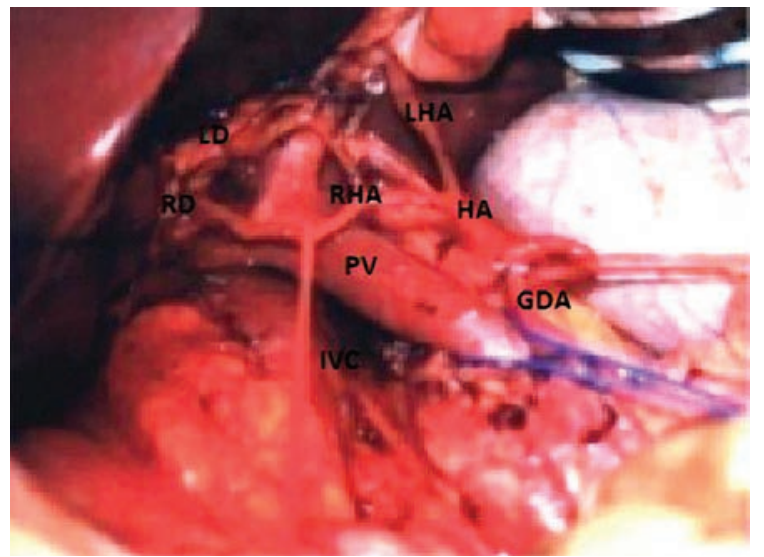

Figure (1): En bloc resection of Klatskin tumour (Bismuth-Corlette type I) shows the proximal right and left ducts ( $R D, L D)$, the gastroduodenal artery (GDA), proper hepatic artery (HA), left and right hepatic arteries (RHA,LHA), portal vein (PV), and inferior vena cava (IVC).
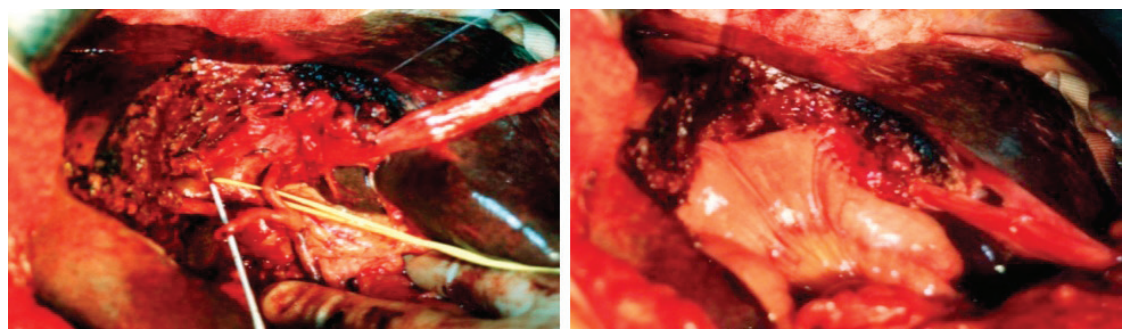

Figure (2): (A) En bolc resection of Klatskin tumor (Bismuth-Corlette type II) with caudate lobectomy. (B) Roux-en-Y hepaticojejunostomy.
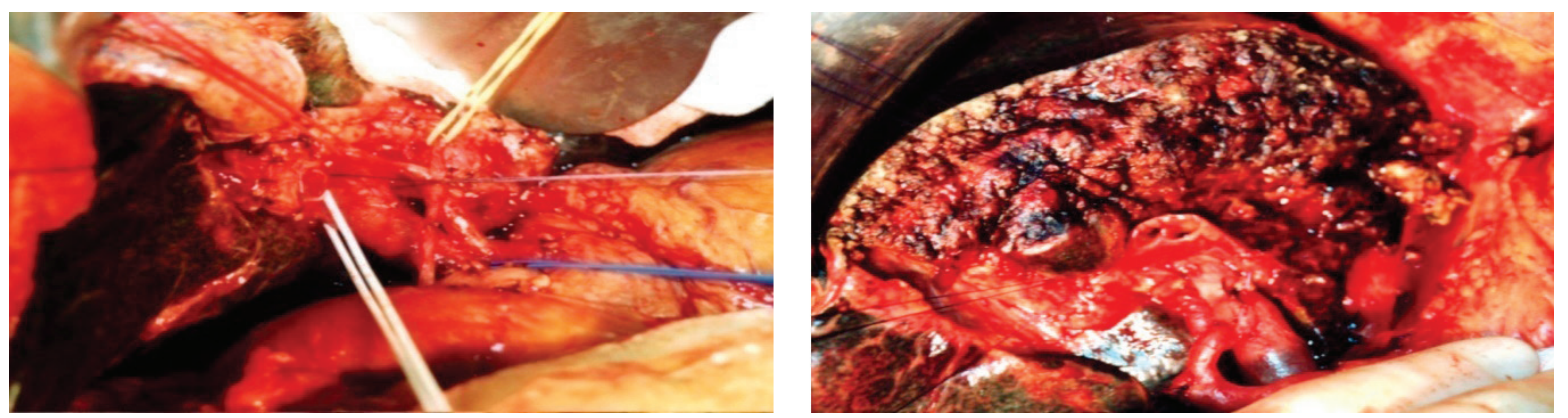

Figure (3): (A, B) Extended left hepatectomy for Klatskin tumor (Bismuth-Corlette type IIIb).
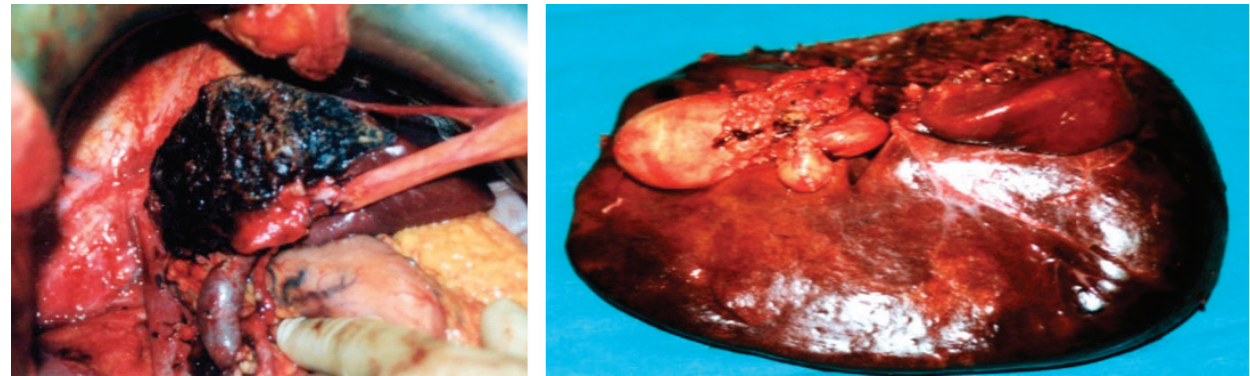

Figure (4): (A, B) Extended right hepatectomy for Klatskin tumor (Bismuth-Corlette type IIIa).

bile duct necessitating more extensive surgery than was expected from the cholangiography alone.

Matsuo $\mathrm{K}$ et $\mathrm{al}^{24}$ provides convincing evidence that a preoperative clinical T staging system, originally proposed by Blumgart and subsequently modified, effectively predicts resectability and likelihood of metastatic 
Table (1): The different presentations encountered in patients with hilar cholangiocarcinoma

\begin{tabular}{|l|l|l|}
\hline Presentation & Number of patients (72) & $\%$ \\
\hline Abnormal liver function tests & 72 & 100 \\
Jaundice & 72 & 100 \\
General malaise & 57 & 79.2 \\
Anorexia & 28 & 38.9 \\
Weight loss & 17 & 23.6 \\
Moderate abdominal pain & 11 & 15.3 \\
\hline
\end{tabular}

Table (2): Patients' age, sex, liver disease, tumor extension and associated co-morbidities.

\begin{tabular}{|l|l|l|}
\hline Patients' data & Number of patients & $\%$ \\
\hline Age (in years) & $41-69$ & \\
Range & $57.8 \pm 10.2$ & \\
Mean \pm SD & 37 & 51.4 \\
$>$ 60 years & 35 & 48.6 \\
$\quad$ < 60 years & 56 & \\
\hline Sex & 16 & 77.8 \\
Male & & 22.2 \\
Female & 58 & \\
\hline Liver disease & 14 & 80.6 \\
Child A & & 19.4 \\
Child B & 65 & \\
Liver cirrhosis & 7 & 90.3 \\
Absent & & 9.7 \\
Present & 36 & \\
\hline Tumor extension & 24 & 50 \\
Bismuth-Corlette type I & 33.3 \\
Bismuth-Corlette type II & 5 & 6.9 \\
Bismuth-Corlette type IIIa & 7 & 9.7 \\
Bismuth-Corlette type IIIb & 7 & \\
\hline Associated co-morbidities & & 23.6 \\
Diabetes Mellitus & 17 & 55.6 \\
Hypertension & 40 & 30.6 \\
Chronic bronchitis & 22 & 52.8 \\
Ischaemic heart disease & 38 & 6.9 \\
Obesity & 5 & \\
\hline
\end{tabular}

disease. In their cohort of 380 patients managed over an 18-year period, approximately $60 \%$ of all patients had unresectable disease, either at presentation or exploration. Although locally advanced disease was an important factor, the most common reason for irresectability was metastatic disease. In their analysis, the resectability rate was $64.3 \%$ in $\mathrm{T} 1$ tumors, $41.3 \%$ in $\mathrm{T} 2$ tumors, and 1.3 (1 patient) in $\mathrm{T} 3$ tumors. Resectability, the probability of an
$\mathrm{R} 0$ resection, and the likelihood of metastatic disease correlated significantly with the clinical $\mathrm{T}$ stage. They found that complete resection with negative margins almost never occurred for T3 tumors but was much more frequent in $\mathrm{T} 1(44.1 \%)$ and $\mathrm{T} 2$ (36.3\%) tumors. The presence of distant metastatic disease increased with more locally advanced and higher clinical $\mathrm{T}$ stage tumors, with $48.7 \%$ of $\mathrm{T} 3$ tumors harboring metastatic 
Table (3): Extent of resection, operative time, intra-operative bleeding, histological grade, lymph node status and hospital stay.

\begin{tabular}{|c|c|c|}
\hline Characteristics & Number of patients & $\%$ \\
\hline $\begin{array}{l}\text { Extent of resection } \\
\text { Bile duct resection + LN dissection } \\
\text { BD resection + LN dissection + caudate lobectomy } \\
\text { Right hepatectomy } \\
\text { Extended right hepatectomy } \\
\text { Left hepatectomy } \\
\text { Extended left hepatectomy }\end{array}$ & $\begin{array}{l}46 \\
14 \\
3 \\
2 \\
4 \\
3\end{array}$ & $\begin{array}{l}63.9 \\
19.4 \\
4.2 \\
2.8 \\
5.6 \\
4.2\end{array}$ \\
\hline $\begin{array}{l}\text { Operative time (in minutes) } \\
\text { Range } \\
\text { Mean }+ \text { SD } \\
\text { Less than } 300 \text { minutes } \\
\text { More than } 300 \text { minutes }\end{array}$ & $\begin{array}{l}180-340 \\
252.7 \pm 47.6 \\
44 \\
28\end{array}$ & $\begin{array}{l}61.1 \\
38.9\end{array}$ \\
\hline $\begin{array}{l}\text { Intraoperative bleeding } \\
\text { Less than } 1000 \mathrm{ml} \\
\text { More than } 1000 \mathrm{ml} \\
\end{array}$ & $\begin{array}{l}57 \\
15 \\
\end{array}$ & $\begin{array}{l}79.2 \\
20.8 \\
\end{array}$ \\
\hline $\begin{array}{l}\text { Blood transfusion } \\
\text { Yes } \\
\text { No }\end{array}$ & $\begin{array}{l}33 \\
39 \\
\end{array}$ & $\begin{array}{l}45.8 \\
54.2\end{array}$ \\
\hline $\begin{array}{l}\text { Histological grade } \\
\text { Well-differentiated } \\
\text { Moderately-differentiated } \\
\text { Poorly-differentiated } \\
\end{array}$ & $\begin{array}{l}41 \\
19 \\
12 \\
\end{array}$ & $\begin{array}{l}56.9 \\
26.4 \\
16.7 \\
\end{array}$ \\
\hline $\begin{array}{l}\text { Number of lymph nodes removed } \\
\text { Range } \\
\text { Median } \\
<12 \\
\geq 12 \\
\end{array}$ & $\begin{array}{l}7-32 \\
17 \\
17 \\
55 \\
\end{array}$ & $\begin{array}{l}23.6 \\
76.4 \\
\end{array}$ \\
\hline $\begin{array}{l}\text { Lymphatic invasion } \\
\text { Negative } \\
\text { Positive } \\
\end{array}$ & $\begin{array}{l}40 \\
32 \\
\end{array}$ & $\begin{array}{l}55.6 \\
44.4\end{array}$ \\
\hline $\begin{array}{l}\text { Lymph node ratio } \\
\qquad 0.2 \\
\quad \geq 0.2\end{array}$ & $\begin{array}{l}57 \\
15\end{array}$ & $\begin{array}{l}79.2 \\
20.8\end{array}$ \\
\hline $\begin{array}{l}\text { Residual disease } \\
\text { No } \\
\text { Yes } \\
\end{array}$ & \begin{tabular}{|l|}
65 \\
7 \\
\end{tabular} & $\begin{array}{l}90.3 \\
9.7 \\
\end{array}$ \\
\hline $\begin{array}{l}\text { Hospital stay (in days) } \\
\text { Range } \\
\text { Mean + SD }\end{array}$ & $\begin{array}{l}6-57 \\
12.7 \pm 4.3\end{array}$ & \\
\hline
\end{tabular}

disease, followed by $34.8 \%$ of $\mathrm{T} 2$ and $19.6 \%$ of T1 lesions.

In an analysis of 225 patients with hilar cholangiocarcinoma, resectability was nearly $60 \%$ in $\mathrm{T} 1$ tumors, $31 \%$ in $\mathrm{T} 2$ tumors, and $0 \%$ in T3 tumors. Survival also decreased with increasing clinical $\mathrm{T}$ stage. ${ }^{23}$

Positron emission tomography has shown a high sensitivity for diagnosing biliary malignancy. Its limitation is that the patients 
Table (4): Postoperative complications.

\begin{tabular}{|l|l|l|}
\hline Complications & Number of patients (72) & $\%$ \\
\hline Hepatorenal failure & 2 & 2.8 \\
Temporarily liver insufficiency & 5 & 6.9 \\
Gastrointestinal haemorrhage & 4 & 5.6 \\
Minor bile leak & 7 & 9.7 \\
Mild wound infection & 11 & 15.3 \\
Prolonged ileus & 2 & 2.8 \\
Intra-abdominal bleeding & 1 & 1.4 \\
Inta-abdominal abscess & 2 & 2.8 \\
\hline
\end{tabular}

with biliary tract infections or inflammatory processes in the biliary tree (as in PSC) can have false positive results. Its best use may be as a diagnostic tool after resection to discover recurrence. ${ }^{31}$

All patients in the present study underwent surgical resection. Forty-six patients underwent bile duct resection with hepaticojejunostomy and regional lymph node dissection. Fourteen patients underwent bile duct resection and lymph node dissection with caudate lobectomy. Three patients underwent right hepatectomy, two patients underwent extended right hepatectomy, four patients underwent left hepatectomy and three patients underwent extended left hepatectomy; all with hilar bile duct resection, caudate lobectomy and regional lymph node dissection. In the 12 patients who underwent major hepatic resections, the residual liver volume was more than $30 \%$ in 10 patients and less than $30 \%$ in two patients. The extended resections that enable a better oncological clearance have become achievable as a result of the major advances in surgical techniques and preoperative and postoperative care, which have reduced morbidity and mortality after major hepatic resection.

The role of preoperative biliary drainage in jaundiced patients remains controversial. Most patients undergo biliary drainage prior to referral for resection, despite the lack of data showing a benefit. The presence of cholangitis mandates biliary decompression, but there is no proof that routine biliary drainage in all patients facilitates resection or reduces postsurgical morbidity. ${ }^{32,33}$ On the contrary, the available data would suggest that biliary stents are associated with greater postoperative infection complications. ${ }^{34}$ Whether major hepatic resection in the face of biliary obstruction is associated with a greater risk of liver failure or other complications remains an open question. ${ }^{35}$ In patients of this study, preoperative biliary stenting is not recommended as it makes dissection more difficult and time-consuming and at the same time decompresses the biliary tree reducing its diameter and making further biliaryenteric anastomosis more difficult.

In the present study, although imaging studies identified many patients with resectable disease, a significant proportion were found to have unresectable disease only at the time of laparotomy. Nearly one third of patients had unresectable tumors at presentation. However, of the remaining patients with potentially resectable lesions, only $50 \%$ underwent resection. As a result, staging laparoscopy has been increasingly used in an effort to reduce the incidence of unnecessary open explorations. In a recent analysis of 56 patients with potentially resectable tumors based on radiological findings, laparoscopy identified unresectable tumors in 14 patients $(25 \%))^{36}$ The yield was significantly higher for patients with clinical T2/T3 tumors (12 of 33, 36\%) compared with those with clinical T1 tumors (2 of 23, $9 \%)$, which is almost certainly related to the higher incidence of metastatic disease in the former group. Laparoscopy detected most patients with peritoneal or liver metastases but failed to detect all locally unresectable tumors. Despite this limitation, however, laparoscopic staging appears to have a role in 
these patients.

Complete resection (R0) was achieved in 65 patients, while 7 patients had incomplete resection. In patients with potentially resectable tumors, there is no doubt that the primary goal of surgery should be a complete resection with histologically negative resection margins (i.e., a R0 resection), which at a minimum requires resection of the extrahepatic biliary apparatus and subhilar lymphadenectomy. However, there are now substantial data to suggest that en bloc partial hepatectomy is also required in most cases. The results of recent studies show a parallel between the number of patients undergoing partial hepatectomy and those having negative resection margins which is a potent predictor of outcome. ${ }^{23,37-46}$ In addition, tumors involving the left hepatic duct almost always involve the main caudate duct and usually require a complete caudate resection as well. ${ }^{47}$ Extensive resections for hilar cholangiocarcinoma have been associated with significant morbidity, and mortality rates, even at high-volume centers, are on the order of 5 to $10 \%$. Infective complications are particularly common and often play a central role in postoperative mortality. 23

In this study, the number of lymph nodes removed was fewer than 12 in 17 patients and equal or more than 12 in 55 patients. The 40 patients with no lymph node metastases had better five-year survival than the 32 patients with lymph node metastases (55\% versus 9.4\%). Fifty-seven patients with LNR less than 0.2 had a five-year survival of $43.9 \%$ in comparison to a five-year survival of $0 \%$ for the 15 patients with LNR equal or more than 0.2. Some authors established a cut-off point of 12 for the number of LNs removed in patients with pancreaticobiliary carcinoma. ${ }^{48,49}$ Similarly, in this study, we adopted the same cut-off point. Numerous studies have demonstrated that patients with lymph node metastases have significantly worse survival rates than patients with node-negative disease. The number of lymph node metastases was an important prognostic factor in patients with extrahepatic cholangiocarcinoma after resection. ${ }^{50,51}$
The lymph node ratio (LNR), defined as the ratio of the number of lymph nodes bearing metastases to the total number of lymph nodes removed, has been demonstrated to be a more important prognostic factor in gastrointestinal carcinomas than the presence of absolute number of lymph node metastasis. ${ }^{52}$ The evaluation of LNR is useful for several reasons. LNR combines data regarding the number of lymph nodes removed with the number of positive nodes, providing information regarding the adequacy of lymphadenectomy. Moreover, LNR is a simple method to stratify patients more accurately via a method that considers both the biology of the disease (number of positive nodes) and the adequacy of lymph nodes dissection (number of excised nodes). Metastatic disease to regional lymph nodes is not uncommon in patients with hilar cholangiocarcinoma. In a recent review of 110 patients, Kitagawa et $\mathrm{al}^{53}$ found that $47 \%$ had no involved nodes, 35\% had regional lymph node metastases, and 17\% had regional and para-aortic node metastases. There was a significant survival difference based on nodal status. Node-negative patients had 3- and 5-year survival rates of 55\% and $30 \%$, respectively, compared with 32 and $14.7 \%$, respectively, for those with regional nodal metastases and $12.3 \%$ in both cases for those with para-aortic node metastases. ${ }^{53}$ Kawai et al ${ }^{48}$ demonstrated that LNR was an important prognostic factor after resection of middle and distal cholangiocarcinoma. Extended lymph node dissection with careful examination for metastases allows for a more accurate evaluation of $\mathrm{LN}$ status and should be performed whenever feasible as it offers a survival advantage in hilar cholangiocarcinoma. In this series, metastatic disease to lymph nodes beyond the hepatoduodenal ligament (celiac, paraaortic, and so on) was a contraindication to resection.

Complete resection was associated with a better survival. However, some patients were not candidates for resection, and these patients were excluded from the study after palliating the biliary obstruction. Percutaneous biliary 
drainage and subsequent placement of a self-expandable metallic endoprosthesis was the preferred approach and was successfully performed in most patients with irresectable HC. Endoscopically placed stents were usually ineffective for proximal biliary obstruction. Biliary stenting was not appropriate in patients with an atrophic lobe as it did not relieve jaundice and was avoided, unless performed to control infection. Patients found to have unresectable, locally advanced tumors at operation were candidates for intrahepatic biliary-enteric bypass. Not infrequently, unresectability was discovered only after an extensive dissection, including transection of the distal bile duct, and biliaryenteric anastomosis was then established to restore continuity. In such cases, the segment III duct was often used, although the right anterior or posterior sectoral hepatic ducts were also used in some cases. In a review of 55 consecutive bypass procedures in patients with malignant hilar obstruction, the authors found segment III bypass yielded the best results, with a 1-year bypass patency rate of $80 \%{ }^{54}$ An advantage of this approach was that the anastomosis was away from the tumor and less susceptible to recurrent obstruction due to disease progression.

There were two perioperative deaths in this series. Death occurred at 7 and 35 days following extended left hepatectomy and right hepatectomy. The cause of death was hepatorenal failure in the two cases. Several groups have explored the possible role of preoperative portal vein embolization; the rationale for this is to induce hypertrophy of the future liver remnant prior to surgery, thereby potentially reducing the risk of postoperative hepatic failure. ${ }^{55-57}$ Although this technique may be of some value, the lack of compelling controlled data makes it difficult to advocate its routine use.

All over 9 patients (12.5) developed recurrence. In this study, the overall estimated survival rate was $51.4 \%$ at three years and $34.7 \%$ at five years. Following resection, 5 -year survival rates were significantly greater in patients who underwent a R0 resection. Furthermore, survival in patients with histologically involved resection margins (R1) was little better than survival in those with unresectable, locally advanced tumors was. Although 5-year survival after a R0 resection was approximately $38.5 \%$ in this series, it is notable that cancer recurrence after 5 years was not uncommon.

This study is limited by the small number of patients. The results of this study will need to be confirmed in a multi-institutional cohort of patients.

\section{Conclusion:}

Cholangiocarcinoma remains a devastating disease. The treatment for cholangiocarcinoma remains a challenge because of the aggressive nature of the disease and the absence of effective treatments besides surgical resection. Most patients have unresectable tumors at the time of diagnosis and have a dismal prognosis. Complete resection (R0) is the only treatment that offers any hope of long-term survival but is possible in few patients. Furthermore, even after resection, disease recurrence is common. Lymph node ratio (LNR) is a powerful independent prognostic factor predicting survival in patients with hilar cholangiocarcinoma after surgical resection. The development of diagnostic modalities (tumour markers, cytology and radiology) are of the utmost importance to identify these patients at an early stage to preserve radical surgery possible. Adjuvant therapy has not been shown to have a role in this disease.

\section{Reference:}

1- Klatskin G: Adenocarcinoma of the hepatic duct at its bifurcation within the porta hepatis. An unusual tumor with distinctive clinical and pathologic features. Am J Med 1965; 38: 241-256.

2- Khan SA, Thomas HC, Davidson BR, TaylorRobinson SD: Cholangiocarcinoma. Lancet 2005; 366: 1303-1314.

3- LazaridisKN, GoresGJ:Cholangiocarcinoma. Gastroenterology 2005; 128: 1655-1667.

4- Curley SA: High malignant biliary tract obstruction. In: Mastery of Surgery. Fischer JE, Bland KI (Editors); Philadelphia: Lippincott Williams \& Wilkins (Publisher); 
$5^{\text {th }}$ edn.; 2007; 1165-1174.

5- Lau SHY, Lau WY: Current therapy of hilar cholangiocarcinoma. Hepatobiliary Pancreat Dis Int 2012; 11: 12-17.

6- Patel T: Cholangiocarcinoma. Nat Clin Pract Gastroenterol Hepatol 2006; 3: 33-42.

7- de Groen PC, Gores GJ, La Russo NF, Gunderson LL, Nagorney DM: Biliary tract cancers. N Engl J Med 1999; 341: 1368-1378.

8- Jarnagin WR, Shoup M: Surgical management of cholangiocarcinoma. Seminars in Liver Disease 2004; 24: 189-199.

9- Weinbren K, Mutum SS: Pathological aspects of cholangiocarcinoma. J Pathol 1983; 139: 217-238.

10- Nakanuma Y, Sato Y, Harada K, et al: Pathological classification of intrahepatic cholangiocarcinoma based on a new concept. World J Hepatol 2010; 2: 419-427.

11- Komuta M, Govaero O, Vandecaveye V, et al: Histological diversity in cholangiocellular carcinoma reflects the different cholangiocyte phenotypes. Hepatology 2012; 55: 1876-1888.

12- Blechacz B, Gores GJ: Cholangiocarcinoma: Advances in pathogenesis, diagnosis, and treatment. Hepatology 2008; 48: 308-321.

13- Nagorney DM, Donohue JH, Farnell MB, et al: Outcome after curative resections of cholangiocarcinoma. Arch Surg 1993; 128: 871-879.

14- Bismuth H, Corlette MB: Intrahepatic cholangioenteric anastomosis in carcinoma of the hilus of the liver. Surg Gynecol Obstet 1975; 140: 170-178.

15- Greene FL, Page PD, Fleming ID, et al: AJCC cancer staging manual. 6th edition. New York: Springer-Verlag; 2002.

16- International Union Against Cancer (UICC): TNM classification of malignant tumors. $6^{\text {th }}$ edn. 2002; 81-89.

17- Chung YU, Kim MJ, Park YN, Lee YH, Choi JY: Staging of extrahepatic cholangiocarcinoma. Eur Radiol 2008; 18: 2182-2195.

18- Jang JY, Kim SW, Park DJ, et al: Actual long-term outcome of extrahepatic bile duct cancer after surgical resection. Ann Surg 2005; 241: 77-84.

19- Todoroki T, Kawamoto T, Koike N, et al: Radical resection of hilar bile duct carcinoma and predictors of survival. Br J Surg 2000; 87: 306-313.

20- Tsao JI, Nimura Y, Kamiya J, Hayakawa N, Kondo S, Nagino M, et al: Management of hilar cholangiocarcinoma: Comparison of an
American and a Japanese experience. Ann Surg 2000; 232: 166-174.

21- Seyama Y, Makuuchi M: Current surgical treatment for bile duct cancer. World $J$ Gastroenterol 2007; 13: 1505-1515.

22- van Gulik TM, Gouma DJ: Changing perspectives in the assessment of resectability of hilar cholangiocarcinoma. Ann Surg Oncol 2007; 14: 1969-1971.

23- Jarnagin WR, Fong Y, DeMatteo RP, et al: Staging, resectability, and outcome in 225 patients with hilar cholangiocarcinoma. Ann Surg 2001; 234: 507-519.

24- Matsuo K, Rocha FG, Ito K, et al: The Blumgart preoperative staging system for hilar cholangiocarcinoma: Analysis of resectability and outcomes in 380 patients. $J$ Am Coll Surg 2012; 215: 343-355.

25- Cheng Q, Luo $\mathrm{X}$, Zhang $\mathrm{B}$, et al: Predictive factors for prognosis of hilar cholangiocarcinoma: Post-resection radiotherapy improves survival. Eur J Surg Oncol 2007; 33: 202-207.

26- Wetter LA, Ring EJ, Pellegrini CA, Way LW: Differential diagnosis of sclerosing cholangiocarcinomas of the common hepatic duct (Klatskin tumors). Am J Surg 1991; 161: $57-62$.

27- Bismuth H, Nakache $\mathrm{R}$, Diamond $\mathrm{T}$ : Management strategies in resection for hilar cholangiocarcinoma. Ann Surg 1992; 215: 31-38.

28- Bold RJ, Goodnight JE Jr: Hilar cholangiocarcinoma: Surgical and endoscopic approaches. Surg Clin North Am 2004; 84: 525-542.

29- Sakamoto E, Nimura Y, Hayakawa N, et al: The pattern of infiltration at the proximal border of hilar bile duct carcinoma: A histologic analysis of 62 resected cases. Ann Surg 1998; 227: 405-411.

30- Abe M, Kondo S, Hirano S, et al: Superficially spreading cholangiocarcinoma. Int J Gastrointest Cancer 2005; 35: 89-94.

31- Friman S: Cholangiocarcinoma: Current treatment options. Scand J Surg 2011; 100: 30-34.

32- Lai EC, Mok FP, Fan ST, et al: Preoperative endoscopic drainage for malignant obstructive jaundice. Br J Surg 1994; 81: 1195-1198.

33- Pitt HA, Gomes AS, Lois JF, et al: Does preoperative percutaneous biliary drainage reduce operative risk or increase hospital cost? Ann Surg 1985; 201: 545-553. 
34- Sarmiento JM, Nagorney DM: Hepatic resection in the treatment of perihilar cholangiocarcinoma. Surg Oncol Clin N Am 2002; 11: 893-908.

35- Klempnauer J, Ridder GJ, von Wasielewski $\mathrm{R}$, et al: Resectional surgery of hilar cholangiocarcinoma: a multivariate analysis of prognostic factors. J Clin Oncol 1997; 15: 947-954.

36- Weber SM, DeMatteo RP, Fong Y, Blumgart LH, Jarnagin WR: Staging laparoscopy in patients with extrahepatic biliary carcinoma. Analysis of 100 patients. Ann Surg 2002; 235: 392-399.

37- Burke EC, Jarnagin WR, Hochwald SN, et al: Hilar cholangiocarcinoma: Patterns of spread, the importance of hepatic resection for curative operation, and a presurgical clinical staging system. Ann Surg 1998; 228 : 385-394.

38- Chen XP, Lau WY, Huang ZY, et al: Extent of liver resection for hilar cholangiocarcinoma. Br J Surg 2009; 96: 1167-1175.

39- Cameron JL, Pitt HA, Zinner MJ, Kaufman SL, Coleman J: Management of proximal cholangiocarcinomas by surgical resection and radiotherapy. Am J Surg 1990; 159: 91-97.

40- Gerhards MF, van Gulik TM, de Wit LT, Obertop H, Gouma DJ: Evaluation of morbidity and mortality after resection for hilar cholangiocarcinoma-a single center experience. Surgery 2000; 127: 395-404.

41- $\mathrm{Su} \mathrm{CH}$, Tsay SH, Wu CC, et al: Factors influencing postoperative morbidity, mortality, and survival after resection for hilar cholangiocarcinoma. Ann Surg 1996; 223: 384-394.

42- Hadjis NS, Blenkharn JI, Alexander N, Benjamin IS, Blumgart LH: Outcome of radical surgery in hilar cholangiocarcinoma. Surgery 1990; 107: 597-604.

43- Dinant S, Gerhards MF, Busch OR, et al: The importance of complete excision of the caudate lobe in resection of hilar cholangiocarcinoma. HPB 2005; 7: 263-267.

44- Neuhaus P, Jonas S, BechsteinWO, et al: Extended resections for hilar cholangiocarcinoma. Ann Surg 1999; 230: 808-818.

45- Kosuge T, Yamamoto J, Shimada K, Yamasaki S, Makuuchi M: Improved surgical results for hilar cholangiocarcinoma with procedures including major hepatic resection. Ann Surg 1999; 230: 663-671.

46- Nimura Y, Hayakawa N, Kamiya J, Kondo
S, Shionoya S: Hepatic segmentectomy with caudate lobe resection for bile duct carcinoma of the hepatic hilus. World J Surg 1990; 14: 535-543.

47- Mizumoto R, Suzuki H: Surgical anatomy of the hepatic hilum with special reference to the caudate lobe. World J Surg 1988; 12: 2-10.

48- Kawai M, Tani M, Kobayashi Y, et al: The ratio between metastatic and examined lymph nodes is an independent prognostic factor for patients with resectable middle and distal bile duct carcinoma. Am J Surg 2010; 199: 447-452.

49- House MG, Gonen M, Jarnagin WR, et al: Prognostic significance of pathologic nodal status in patients with resected pancreatic cancer. J Gastrointest Surg 2007; 11:1549-1555.

50- Sasaki R, Takahashi M, Funato O, et al: Prognostic significance of lymph node involvement in middle and distal bile duct cancer. Surgery 2001; 129: 677-683.

51- Hong SM, Cho H, Lee OJ, Ro JY: The number of metastatic lymph nodes in extrahepatic bile duct carcinoma as a prognostic factor. Am J Surg Pathol 2005; 29: 1177-1183.

52- Pawlik TM, Gleisner AL, Cameron JL, et al: Prognostic relevance of lymph node ratio following pancreaticoduodenectomy for pancreatic cancer. Surgery 2007; 141: 610-618.

53- Kitagawa Y, Nagino M, Kamiya $J$, et al: Lymph node metastasis from hilar cholangiocarcinoma: Audit of 110 patients who underwent regional and para aortic nodedissection. Ann Surg 2001;233:385-392.

54- Jarnagin WR, Burke E, Powers C, Fong Y, Blumgart LH: Intrahepatic biliary enteric bypass provides effective palliation in selected patients with malignant obstruction at the hepatic duct confluence. Am J Surg 1998; 175: 453-460.

55- Kawasaki S, Makuuchi M, Miyagawa S, Kakazu T: Radical operation after portal embolization for tumor of hilar bile duct. $J$ Am Coll Surg 1994; 178:480-486.

56- Makuuchi M, Thai BL, Takayasu K, et al: Preoperative portal embolization to increase safety of major hepatectomy for hilar bile duct carcinoma: A preliminary report. Surgery 1990; 107: 521-527.

57- Nagino M, Nimura Y, Kamiya J, et al: Right or left trisegment portal vein embolization before hepatic trisegmentectomy for hilar bile duct carcinoma. Surgery 1995; 117: 677-681. 